\title{
Neuer Thrombozytenaggregationshemmer
}

\section{Längere Lebenserwartung, kein erhöhtes Blutungsrisiko}

Die kardiovaskuläre Mortalität lässt sich bei Patienten mit akutem Koronarsyndrom mit dem Thrombozytenaggregationshemmer Ticagrelor signifikant stärker senken als mit Clopidogrel. Dieser Überlebensvorteil wird nicht mit einem Anstieg schwerer Blutungen erkauft. Das sind die wichtigsten Ergebnisse der PLATO-Studie.

Neuen Analysen zufolge zeigen sich die Vorzüge von Ticagrelor bei allen untersuchten Subgruppen von Patienten mit unterschiedlichen Grunderkrankungen. Das berichtete Prof. Hugo A. Katus aus Heidelberg bei der Tagung der Deutschen Gesellschaft für Kardiologie.

\section{Weniger Komplikationen als mit Clopidogrel}

An der PLATO-Studie haben mehr als 18500 Patienten mit einem akuten Koronarsyndrom teilgenommen. Zur dualen Thrombozytenhemmung erhielten sie ASS entweder plus Ticagrelor oder plus Clopidogrel, und zwar für die Dauer von zwölf Monaten. In diesem Zeitraum trat der primäre Endpunkt (vaskulärer Tod, Herzinfarkt oder Schlaganfall) unter Ticagrelor bei 9,8\% der Patienten und damit signifikant seltener auf als unter Clopidogrel (11,7\%, relative Risikoreduktion 16\%).

Eine weitere Untersuchung zu Ticagrelor soll noch in 2010 starten: Die PEGASUS-TIMI-54-Studie mit insgesamt
21000 Patienten. In dieser Studie wird die langfristige Wirksamkeit und Sicherheit von Ticagrelor bei Patienten untersucht, bei denen der Herzinfarkt bereits ein bis drei Jahre zurückliegt und die ein stark erhöhtes Risiko für weitere Ereignisse aufweisen.

Die Europäische Zulassungsbehörde (EMA) hat Ticagrelor (Brilique ${ }^{\mathrm{TM}}$ ) am 6. Dezember 2010 zur Behandlung von Patienten mit akutem Koronarsyndrom zugelassen.

(rk) II

II Symposium, DGK-Herbsttagung, Nürnberg, 7. Oktober 2010 (Veranstalter: AstraZeneca)

\section{Erster Fortschritt seit 20 Jahren in der Herzinsuffizienztherapie Neues Therapieprinzip ist als Add-on-Therapie wirksam}

Die SHIFT-Studie hatte ergeben, dass bei Herzinsuffizienzpatienten mit einer Herzfrequenz über 70 Schlägen pro Minute, der $\mathrm{I}_{\mathrm{f}}$-Kanalhemmer Ivabradin als Zusatztherapie zum Betablocker statistisch signifikant die Zahl der Hospitalisierungen und der kardiovaskulären Ereignisse um relative $18 \%$ senkt.

Prof. Michael Böhm, Homburg/Saar, wies darauf hin, dass es sich bei der SHIFTStudie mit 6558 Patienten um die bisher größte Einzelstudie zur Herzinsuffizienz überhaupt handelt. Sie lieferte vor allem die wissenschaftliche Erkenntnis, dass die hohe Herzfrequenz nicht nur ein Risikomarker, sondern auch ein Risikofaktor sei. Im Schnitt hatte die Ivabradinbehandlung die Herzfrequenz in der SHIFT-Studie um elf Schläge pro Minute gesenkt - ausgehend von etwa 80 Schlägen pro Minute. Wie Böhm erklärte, könne das insuffiziente Herz bei verringertem Puls eine längere Füllung und eine verbesserte Kontraktion mit größerer Auswurfleistung realisieren.

Außerdem gebe es eine starke „UseDependance“. Das bedeutet: „Ist die Frequenz niedrig, senkt Ivabradin wenig. Ist die Frequenz hoch, senkt es viel." Prof. Karl Werdan, Halle, erläuterte, dass in der Studie 90\% der Patienten einen Betablocker erhielten, davon die Hälfte mit zumindest halber Dosierung. Das entspreche der Klinikrealität in Deutschland. „Es ist schwierig, bei Herzinsuffizienz hohe Betablockerdosierungen zu erreichen.“ Und: „Die Compliance für die hohen Betablockerdosierungen ist schlecht, auch aufgrund von Nebenwirkungen."

Werdan verband diese Feststellung mit der Hoffnung, dass das Medikament möglichst schnell auch für diese neue Indikation zugelassen wird. Phosphene im Auge als unerwünschte Wirkungen unter Iva- bradin seien selten und reversibel. Beide Kardiologen stellten heraus, dass es sich bei der Substanz um das erste neue Therapieprinzip bei Herzinsuffizienz nach der Einführung der ACE-Hemmer vor 20 Jahren handele.

Auch KHK-Patienten, die häufig eine Herzinsuffizienz entwickeln, profitierten bei erhöhter Herzfrequenz von der Substanz, wie eine Untergruppe der BEAUTIFULStudie ergeben hatte, so Werdan. Die in klinischen Leitlinien empfohlene Ruheherzfrequenz von 55 bis 60 Schlägen bei Herzinsuffizienz könne nun bei wesentlich mehr Patienten erreicht werden. Die Zahl der zu behandelnden Patienten (NNT) für ein Jahr, um ein kardiovaskuläres Ereignis zu verhindern, betrug in SHIFT 26.

(rk) I

II Pressegespräch, München, 13. September 2010 (Veranstalter: Servier) 